\title{
MULTIPARAMETER SYSTEM FOR MONITORING THE STATE OF URBANOSPHERUM BASED ON MULTIVENDOR DEVICES OF DATA COLLECTION
}

\author{
Aleksandr Pilipenko ${ }^{1}$, Anastasiia Pilipenko ${ }^{2}$ \\ ${ }^{1}$ Orel State university named after I.S. Turgenev, 302026, Orel, Russia \\ ${ }^{2}$ Proton-Electrotex, 302040, Orel, Russia
}

\begin{abstract}
The authors explain the problem of partitioning of the control object on the controlled elements, and give a description of the mathematical apparatus. The authors propose different types of portable devices for collecting information about the state of urbanospherum on the basis of different hardware and software platforms that combine integrated information system. In the work the presented algorithms and block diagrams of data collection devices are based on the controller NI MyRIO, micro-computer Raspberry Pi and Arduino microcontroller system. The authors explain the approach to the optimization of systems of management of urban resources and processes with the using of systems condition monitoring of urbanospherum.
\end{abstract}

\section{Introduction}

The city is a multi-parameter system consisting of a large number of resources which are various according to the purpose and the content, and which are integrated by the processes taking place between them. These are industrial enterprises and public organizations, institutions of health and education, utilities and service sector enterprises, construction and energy companies, etc., successful functioning of which depends directly on transportation infrastructure, the condition of linear objects and buildings, from the management of life supporting processes, security environment and, primarily, from the human condition from biosphere compatibility of nature and socio-technical structures. At present, there is no structured data about the interaction processes between participants in the life of the city, their impact on the urban environment. Information about the objects and the relationships between them are completely different from the point of view of the format, the synchronization in time, the methods of processing, storage and transmission. These factors do not allow timely to track and to provide ecological, technogenic safety of the city and the rapid response of special services to emergency situations.

\footnotetext{
${ }^{1}$ Corresponding author: a@pilipenko.info
} 


\section{Concept}

Markets of public safety in urbanized areas that include fire safety, water safety, public security, traffic safety, environmental and industrial safety were analyzed. It was revealed that the system of city management and urban farms could be described by quantitative and qualitative indicators of biosphere compatibility of different structures. The objective function of the system of city management should strive for minimum cost, minimum time of decision-making and the minimum number of emergency situations. The very same objective function of the management system is multivariable, which depends on many parameters of the urban environment, the collection of which is possible due to monitoring systems. The development of multiparameter systems of monitoring the condition of urban processes and resources and integrated implementation of information systems in all of the management, administration and emergency services will not only create a system of quality management of a city with elements of feedback, but also give the opportunity to assess the work quality of city structures.

The goals of the development of multiparameter monitoring systems of the urbanospherum state are the following:

1) the increase of security and the improvement of the quality of life of the city population;

2) the increase of city management efficiency;

3) the transition from information systems that use different databases to a single integrated information environment that provides automated support of processes of activity of departments of the Ministry of Emergency Situations (MES), authorities, organizations and enterprises of the city, without losing any existing data;

4) the reduction in time-consuming routine operations related to the exchange of information performed at all stages of technological processes;

5) the improvement of the validity of obtained, processed, and stored information used in the process of activity of divisions of the MES, authorities, organizations and enterprises of the city, by centralizing its storage, regulation of use;

6) the reduction of terms and improvement of quality of technological processes, as well as the preparation and registration of documents;

7) technological support for collecting and processing the accounting information, automation of reporting;

8) the creation of a tool of automated support for management decision-making on the basic directions of activity of divisions of bodies of the MES, the authorities, organizations and enterprises of the city.

The criterion of achieving the set goals is the organization of a single information space which ensures the complete implementation of the goals of creating a multiparameter system of monitoring the state of the urbanospherum, the ability of the divisions of the MES, authorities, organizations and enterprises to perform their functions with the least time cost, to improve the quality of life and to provide comprehensive security of the city population.

The urbanospherum refers to some part or parts of the urban environment; or the environment or conditions of existence, similar to the conditions of existence in the urban environment; or the environment, from resource-dependent urban environments [1]. A distinctive feature of the urbanospherum is the presence of a single purpose of its functioning. For example, the urban water supply system is understood by all as a system of single purpose functioning, it is controllable, and thus it is the urbanospherum. However, autonomous regional water supply system, being a part of a citywide water supply system, also has a common goal and also is controllable, that it is the urbanospherum. Several urban 
spheres can be located on the same territory and use the same resources, the elements of the management system and even be located completely on the same material objects.

The authors propose a multi-parameter system of monitoring the urbanospherum condition which includes:

- Application subsystems are a set of software means and their documentation designed to solve relatively narrow classes of problems in specific subject areas designed to a specific customer, which are closely related to each other for organizational, informational, technological or other grounds.

- Providing subsystems are a set of program-technical means, intended for organization of interaction between different application subsystems among themselves and between the data collection devices.

- Data collection system is a set of software and hardware means for collecting, processing and transmitting information about the state of the urban environment into providing subsystems.

Examples of application subsystems are "System-wide sectoral data bank", "Normativereference information", "Geoinformation system", "Ecoinformation system", "Monitoring of the city resources", "Monitoring of urban processes", etc. All of these subsystems can be developed in any programming environment on any programming languages, but united by a common database. However, the authors believe that the graphical programming environment LabVIEW from National Instruments is the most appropriate environment for the development of automated systems. The biggest advantage of LabVIEW environment is the large number of developers around the world. Almost all universities that implement training direction 15.03.04 "Automation of technological processes and productions" have LabVIEW in their educational program. A large number of developers lead to a large number of examples, libraries and modules to work with almost any device from the microcontroller system Arduino to the programmable quadcopter Ar Drone.

Let us consider the development of the most common subsystems and their elements. Figure 1 shows a screenshot of a window application subsystem device "Management of the data collection devices" in the mode of recommendations on the placement of data collection devices. The task of this subsystem is to calculate the optimal location of devices for data collection depending on resources and priority of the borders of the urbanospherum.

The use of triangulation [2] reduces the problem of state analysis of city resources and processes to the finite element method, where many smaller urban spheres are controlled by different types of data collection devices, and the recommendation on the necessary controlling actions affecting the urban sphere as a whole is based on these data. The operator selects the number of active data collection devices, which would mean the number of elements in the triangulation. The number of priority resources of the city shall be selected using the unit Radio Button, which is connected to the element Tab Control and Case Structure, on which input field coordinate city resources are located, and this allows displaying and using the selected number of data entry fields. The coordinates of the triangulation points are set manually or filled from the database automatically, then using the API functions the territory map is loaded with the site www.google.ru/maps/, on which the points and the results of triangulation are mapped with which with the help of virtual devices of NI Vision module. 


\section{The recommended placement of data collection devices}
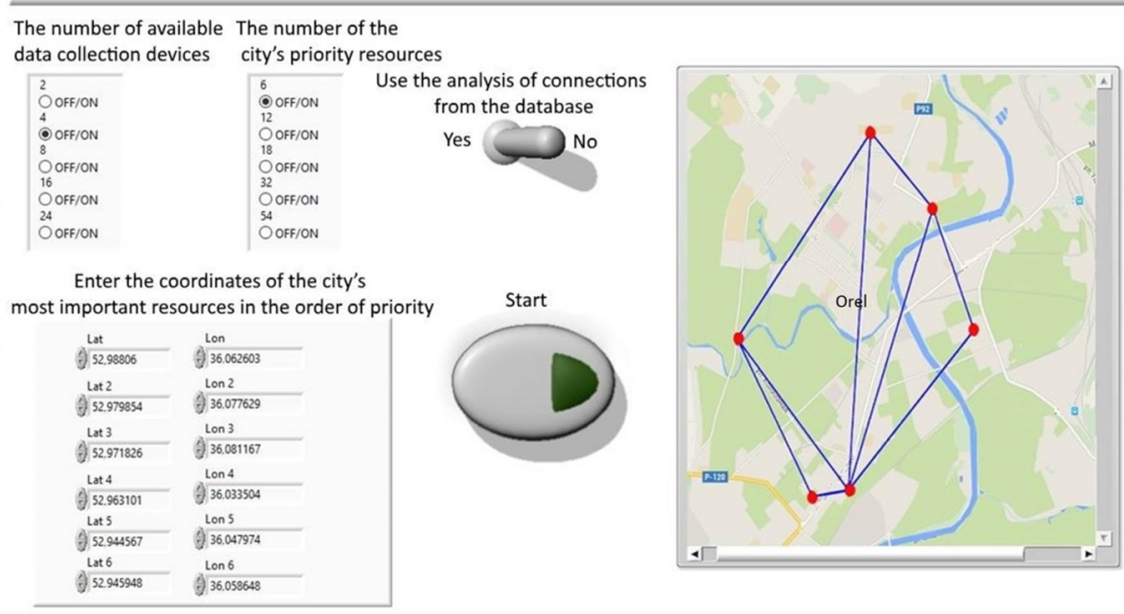

Figure 1. The picture of the window of the application subsystem in the mode of the placement of data collection devices.

The mathematical apparatus for constructing the convex hull of the points (Figure 2) and the optimal triangulation by a greedy algorithm is partly implemented in MathCAD [3, 4], and partly in LabVIEW. The connection of MathCAD and other mathematical software with programming environments are usually implemented through the development of own dll libraries, but the LabVIEW community has already been implemented in MathcadLabVIEW Integration Wizard with the developed library MCVI Wizard.llb.

The application subsystem is connected to the MS SQL database, which contains information about major businesses, emergency services, administrative structures and the processes taking place between them. The function of linkage analysis allows to refine the triangulation and to build it not with the aim of obtaining the minimum sum of the lengths of edges, but with the aim of connecting resources among themselves according to links laid down in the database. The flowchart of the subprogramme responsible for the interaction with the database using the NI LabVIEW Base Connectivity Toolkit Data [5] is presented in Figure 3.

Data collection devices can be completely different both at the level of hardware, and at the level of programming languages and environments. Let us consider several types of data collection devices:

1. Portable devices for collecting information on the state of the personal urbanospherum.

2. Stationary devices for collecting data about an urban environment that is installed on public transport and pillars. The point of data collection will have an extensive array of sensors and components for photo-video-recording. 


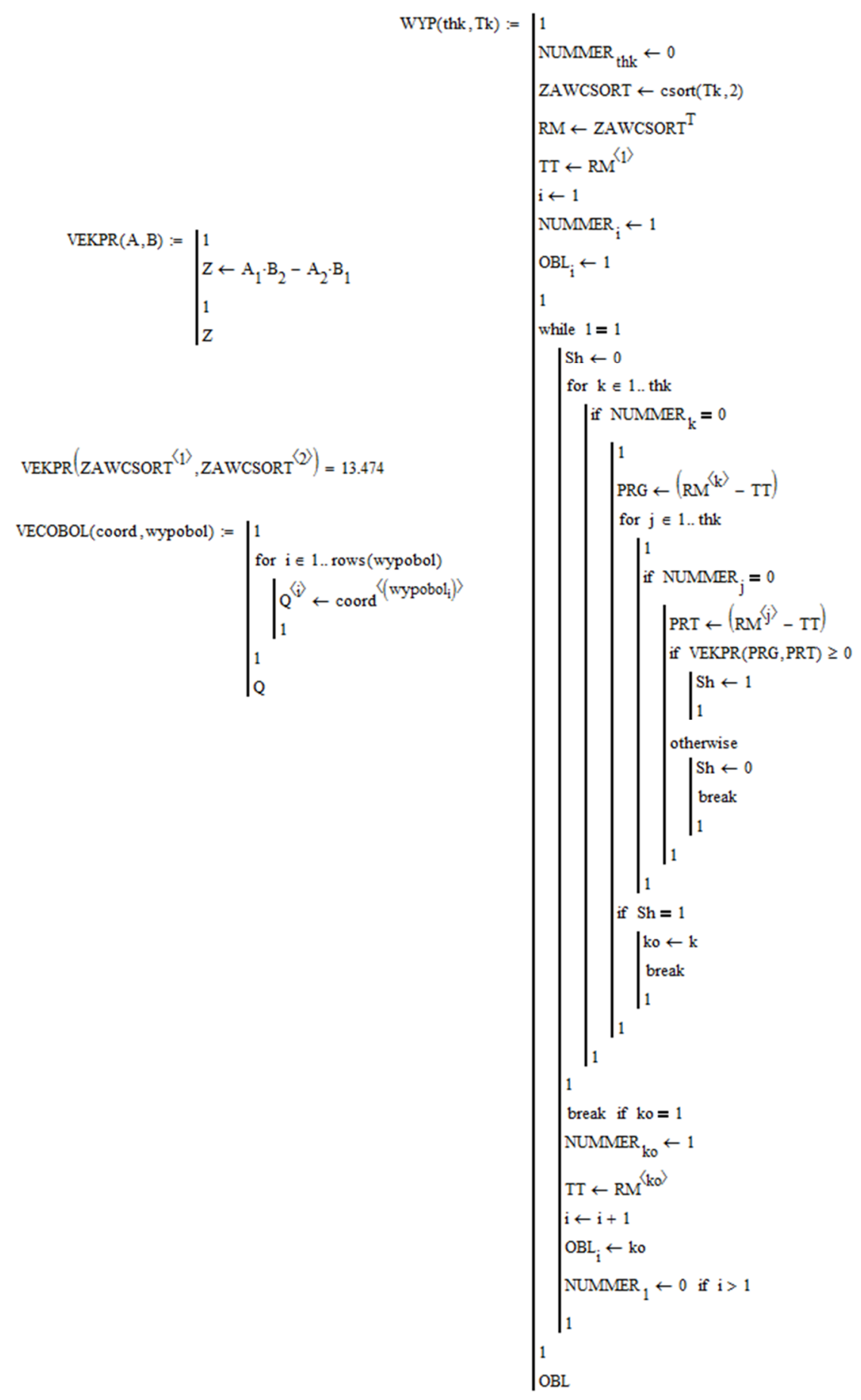

Figure 2. The construction of the convex hull of the set of points for triangulation in MathCAD. 


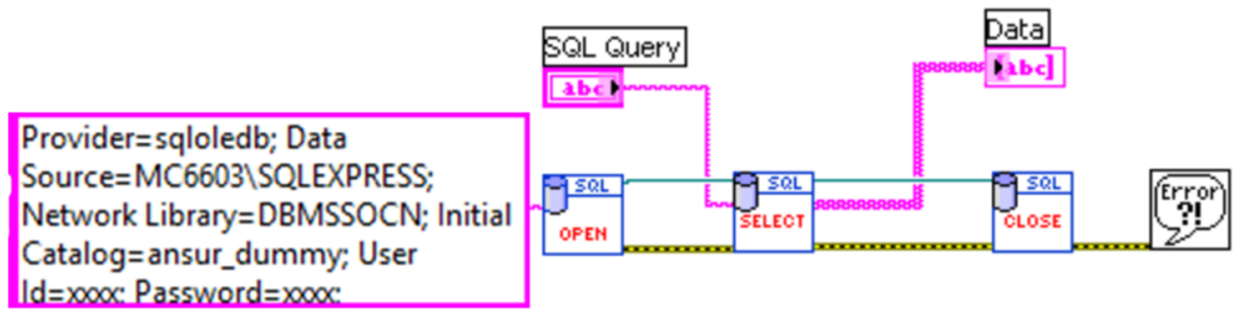

Figure 3. The flowchart of the subprogram responsible for the interaction with the database.

The personal urbanospherum, being a special case of the urbanospherum, retains all its properties. Everything that a particular person living in the urbanized area communicates with can apply to this concept. The development of the wearable device for collecting information about the human condition is performed on two different systems and programming languages:

1. The laboratory version of the device [6]. The device is based on a chip Arduino Lilypad on the base of the microcontroller AVR by connecting the chipset and sensors to it. The programming language is $\mathrm{C}++$, the programming environment is Arduino IDE. The small size of electronic components allows implementing the system in the form of "the smart clothing". The link with other system modules is organized by the wireless channel of the Zigbee data.

2. The casual version of the device. The information gathering device is based on a ready product Samsung Gear S, which has a completely open operating system Tizen, which gives the ability to change even the core of the system. The sensor pulse, accelerometer, gyroscope, GPS, microphone, in a stylish designer case with optimized power consumption. Communication interfaces are GPRS, Bluetooth and Wi-Fi, the programming is supported in JavaScript

The devices for gathering information about the human environment are also presented in two variations:

1. A stationary device that is installed on public transport and pillars. Information gathering device about the external environment is on the basis of NI MyRIO [6], which is based on a Cortex ARM 9 and has a built-in accelerometer, gyroscope, Wi-Fi and plenty of ports for connecting sensors and cameras. The programming environment from the manufacturer NI LabVIEW is a set of libraries and modules allowing to solve tasks of any complexity including the collection and processing of images.

2 . The mobile version of the device is based on a compact micro-computer Raspberry Pi 2, which also runs on the Cortex ARM 9 and has a USB. Inputs, GPIO ports and a connector for connecting the camera. Connecting an additional extension board of GPIO ports allowed to increase their number from 17 to 30 and to add analog inputs. The device weight with all connected sensors is not more than 300 grams. The operating system of the micro-computer is Linux and Windows, the recommended programming language is Python.

Figure 4 shows a conceptual diagram of data collection devices reflecting connections between hardware components of data collection devices. The connection between data collection devices and application subsystems is implemented through wireless and wired technologies, and it doesn't have to be popular, but energy-consuming data transfer channels GPRS and Wi-Fi [7]. Almost all devices work with all receivers/transmitters Zigbee, which allow transmitting information at a distance of from 10 to 1500 meters with high energy efficiency and with the support the cryptographic protection. The algorithm of the work of the subprogramme of a message receiver over a wireless channel Zigbee is shown in Figure 5. 


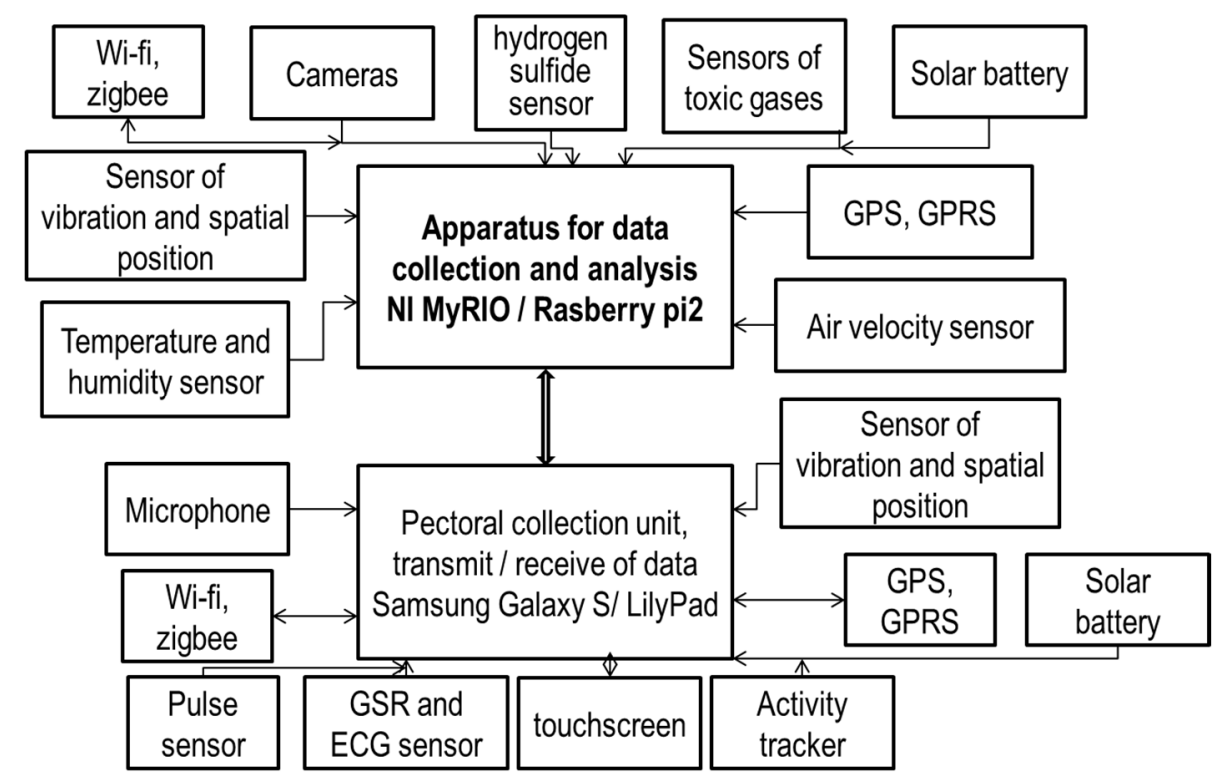

Figure 4. Conceptual diagram of the data collection devices.

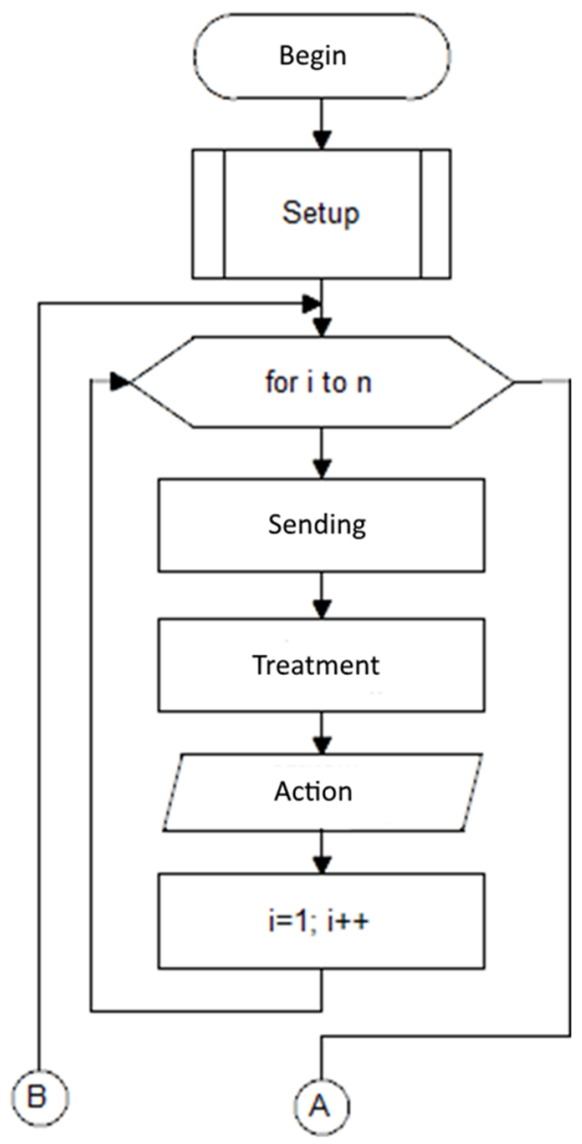




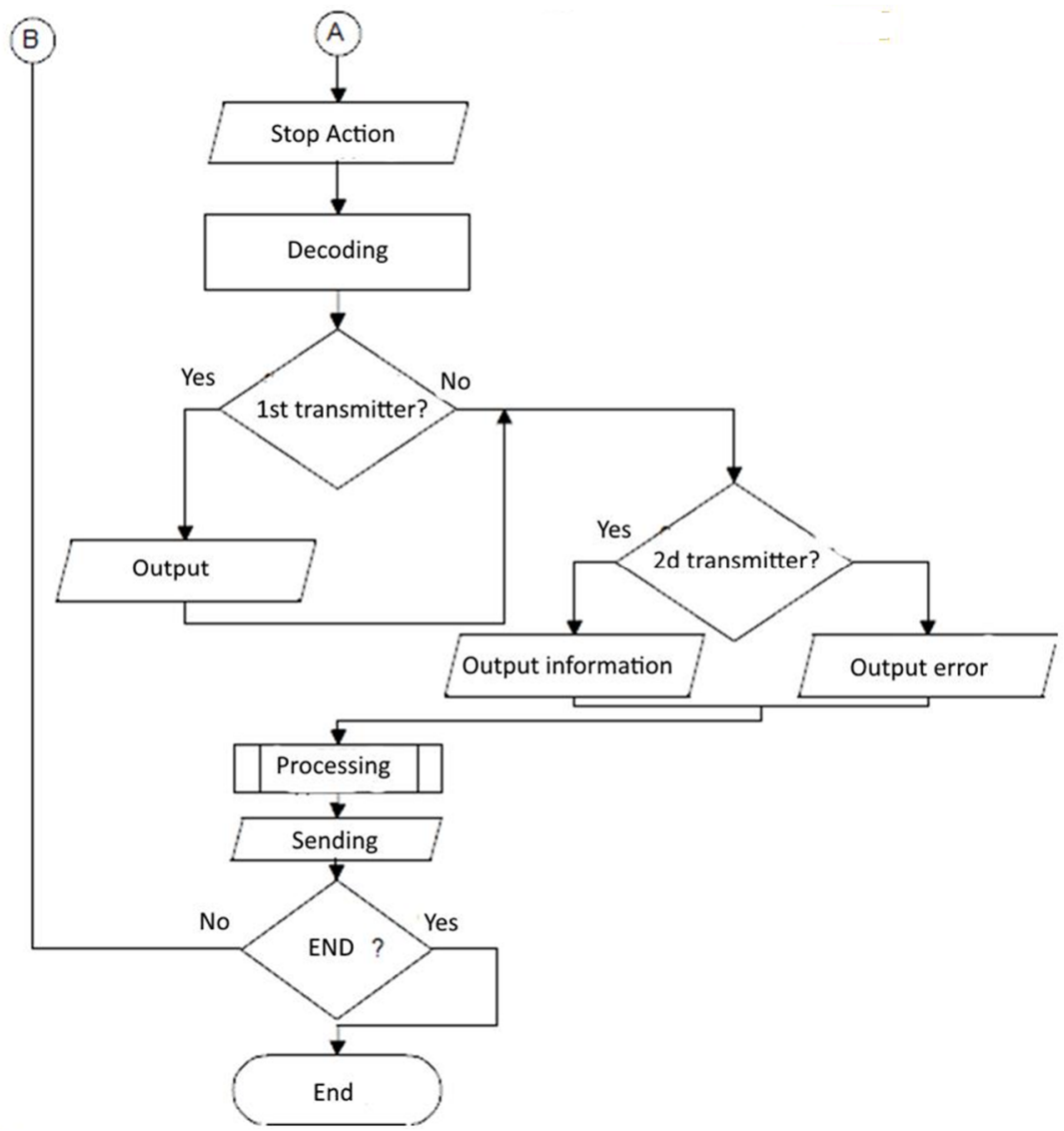

Figure 5. The receiver algorithm.

Stationary devices have great performance and accuracy of measurements, and also allow the connection of devices of photo and video- fixing (cameras) to collect and to display operational data on incidents. The programme structure of the computer vision is presented in Figure 6.

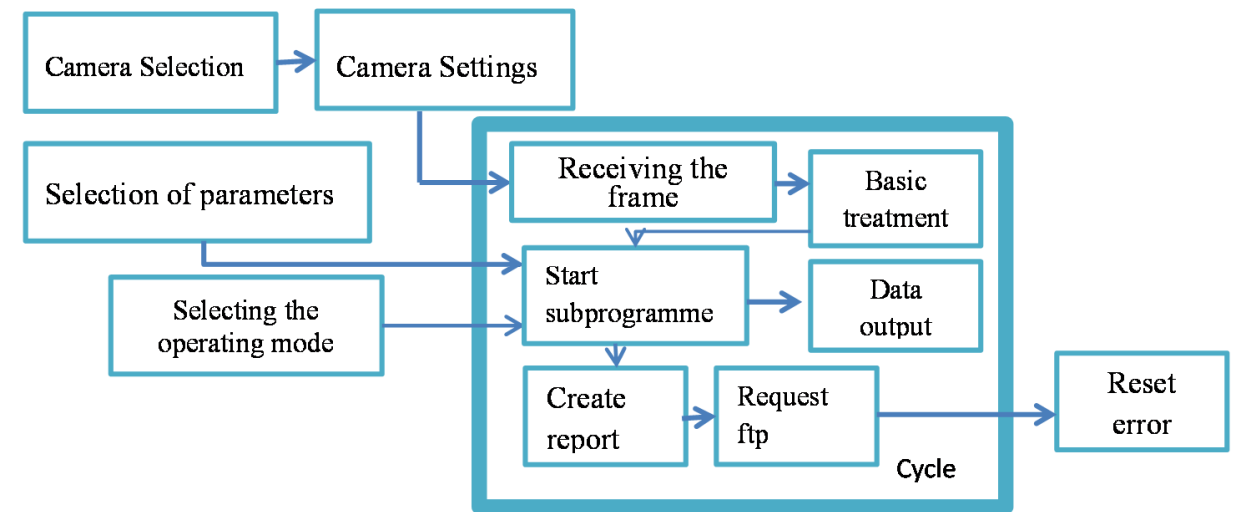

Figure 6. Structural scheme of the system under development. 
The algorithms for collecting photo-video data, initial processing and their transfer to the FTP server have been developed and tested (Figure 7) [8]. The developed subsystems allow to determine fires and ignitions based on the algorithms for the analysis of the illumination intensity, and to determine the speed of the vehicle, the entering vehicle registration, the analysis of speed and density of traffic flows, the definition of a traffic collision based on the algorithms of the detection and comparison of images. The whole effect is that the system deducts a few frames out of each other, and when the same code is subtracted from the code of the pixel, 0 is obtained that the code system of colors is equal to zero, i.e. if there is a moving object, in the array of pixels, the photos will be non-zero pixels.

To determine the speed it is enough to write a code that divides the size of the road in front of the camera for the time that the car overcomes it. It is rather easy to define time knowing the number of images that have nonzero pixels and the number of images the camera shoots per unit of time.

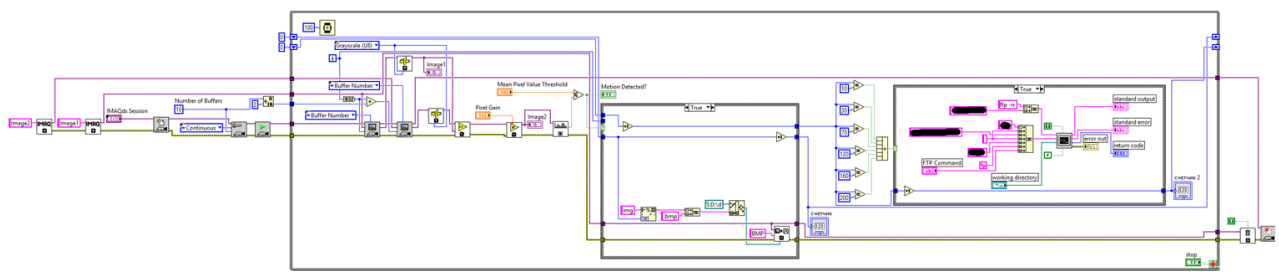

Figure 7. The block-diagram for collecting, processing and transfer of images via FTP.

\section{Conclusion}

The developed multi-parameter monitoring system of the state of the urbanospherum is a modular system that allows:

- to work out application subsystems for specific structural divisions;

- to develop and to implement providing subsystems to combine existing information systems;

- to obtain systematic and current data on the state of urban processes;

- to develop methods for qualitative and quantitative analysis of the security environment of the urbanized territories;

- to obtain quantitative estimates of the resources and processes of the urban environment;

- to identify the dynamic links between natural and socio-technical structures of the city that have the greatest influence on the development of biosphere compatibility of city safety;

- to display to the user the processes taking place in a city under specified conditions, to establish the reasons of occurrence of those or other situations.

Further development of this complex of systems will allow:

- to predict the possible consequences of control actions and prevent risks to the life and health of people;

- to produce qualitative (expert) analysis of the interaction between natural and sociotechnical structures of a city with the desired degree of detail;

- to provide a sufficiently detailed simulation of the interaction process of the MES, authorities, organizations and businesses, residents of a city in the conditions of the current time;

- to implement the automated decision-making support into the management of urban resources and processes. 
As a result of theoretical studies the mathematical model of construction of a convex hull and triangulation, which is now used to split the town in parts, and will continue to be used in the implementation of projects related to GLONASS and persons' identification. In the course of performing applied research we developed a device that raised the interest of many industrial enterprises - a device analyzing the state of the operator and his personal urbanospherum, and it is a portable individual unit to carry out monitoring of the movement of staff on the workshop floor, deviations in person's psychophysiological state and deviations in the parameters of aero-gas-dynamic control. Such systems allow not only to reduce the response time to an emergency situation, but also to optimize production processes and enterprise management system as a whole. The developed mathematical model of triangulation will be checked and analyzed by using the device of collection and analysis of data about urbanospherum. All developments are carried out with the support of the Grant of the President of the Russian Federation and regularly registered in Rospatent and published in peer-reviewed journals included in the list recommended by the Higher Attestation Commission.

\section{References}

[1] V.G. Abashin, A.V. Pilipenko, Proceedings of the International Conference on Intelligent Information Systems (IIS2013) E 9, 196 (2013)

[2] D.-T. Lee, B. J. Schachter, International Journal of Computer \& Information Sciences 9, 219 (1980) doi: 10.1007/BF00977785

[3] Visualization program for solving the problem of finding a triangulation: the registration certificate of the software for the computer. The Russian Federation. No. 2016611364; declared 03.12.15; published 01.02.16

[4] The program of debugging the problem of finding a triangulation by the method of conjugate faces of barrier and penalty functions: The registration certificate of the software for the computer. The Russian Federation. No. 2016610841; declared 03.12.15; published 20.01.16

[5] K. Elmansouri, R. Latif, B. Nassiri, F. M. R. Maoulainine, Journal of medical systems 38 , (2014) doi: 10.1007/s10916-014-0039-8

[6] R. Lynn, A. Chen, S. Locks, C. Nath, T. Kurfess, IFIP Advances in Information and Communication Technology 459, 27 (2015) doi: 10.1007/978-3-319-22756-6_4

[7] Software for automation of the premises on the Arduino platform: the registration certificate of the software for the computer. The Russian Federation. No. 2015662105; declared 28.09.2015; published 17.11.2015

[8] Multi-function control system based on machine vision: the registration certificate of the software for the computer. The Russian Federation. No. 2013613901; declared 04.03.2013; published 18.04.2013 